\title{
Análisis panbiogeográfico de algunas Cactaceae del Ecuador
}

\section{Panbiogeographic analysis of some Cactaceae from Ecuador}

\author{
Christian R. Loaiza S. ${ }^{*} \&$ Juan J. Morrone ${ }^{2}$ \\ ${ }^{1}$ Instituto de Ecología, Unidad de Ecología y Fisiología Vegetal, Universidad Técnica Particular de Loja, Loja, Ecuador. \\ ${ }^{2}$ Museo de Zoología "Alfonso L. Herrera", Departamento de Biología Evolutiva, Facultad de Ciencias, Universidad Nacional \\ Autónoma de México (UNAM), Apartado postal 70-399, 04510 México, D.F., México. \\ *crloaiza@utpl.edu.ec; juanmorrone2001@yahoo.com.mx.
}

\begin{abstract}
RESUMEN
Se analizó la distribución geográfica de 12 especies de cactáceas incluidas dentro de cinco géneros utilizando un enfoque panbiogeográfico, con el fin de identificar los patrones biogeográficos de este grupo y contribuir al estudio de las relaciones biogeográficas de la flora del Ecuador. Las especies seleccionadas se distribuyen principalmente en los bosques secos del norte de Perú y sur del Ecuador. Mediante el análisis de trazos, se identificaron tres trazos generalizados (costero seco, interandino del centro-norte e interandino del sur) y un nodo en la confluencia del segundo y tercer trazos a nivel de la provincia biogeográfica del Occidente de Ecuador.
\end{abstract}

Palabras clave: Cactaceae, panbiogeografía, trazos generalizados, bosques secos, distribución.

\begin{abstract}
The geographical distributions of 12 species of Cactaceae assigned to five genera were analyzed using a panbiogeographic approach to identify their biogeographic patterns and contribute to the study of the biogeographical relationships of the flora from Ecuador. The selected species are distributed mainly in the dry forests of northern Peru and southern Ecuador. Based on the track analysis, three generalized tracks (dry coastal, central-northern interandean and southern interandean) and one node were identified, the latter in the overlap of the second and third tracks at the biogeographic province Western Ecuador.
\end{abstract}

KeYwords: Cactaceae, panbiogeography, generalized tracks, dry forests, distribution.

\section{INTRODUCCIÓN}

La familia Cactaceae es endémica de América. Su distribución comprende todo el continente, con excepción de las zonas altoandinas. Desde una perspectiva dispersalista, los especialistas han considerado tradicionalmente a la zona tropical seca de América del Sur como el probable centro de origen de la familia (Bravo 1978). A nivel de Ecuador, esta familia se encuentra representada por 16 géneros y 43 especies nativas (Loaiza et al. 2009), de las cuales 13 especies son endémicas del país, sin incluir las Islas Galápagos (Madsen 1989, 2002, Ulloa \& Neill 2005, Valencia et al. 2000). Entre las zonas con mayor endemismo y diversidad, se considera al sur del Ecuador (El Oro, Loja y Zamora Chinchipe). Esta región concentra la mayor diversidad de especies, con 13 géneros $(81,3 \%)$ y 28 especies (58,3\%) (Madsen 2002, Loaiza et al. 2009). Otras provincias que también se caracterizan por su alta diversidad son Azuay, Cañar y Chimborazo, con algunos taxones igualmente endémicos como Cleistocactus leonensis Madsen, Armatocereus godingianus (Britton \& Rose) Backeb. ex E. Salisb., Opuntia soederstromiana Britton \& Rose y Espostoa frutescens Madsen (Britton \& Rose 1920, Madsen 1989). Debido a su carácter endémico y a la presencia de ciertas características primitivas como las espinas en flores y frutos, es probable que los géneros Armatocereus y Browningia representen relictos de una flora xerofítica que habría estado distribuida más ampliamente durante el período glacial, debido a que las condiciones climáticas en aquel entonces eran mucho más áridas a nivel del continente (Madsen 2000).

Los estudios realizados sobre las cactáceas del Ecuador han sido abordados en su mayoría bajo un enfoque taxonómico y distribucional, sin analizar detenidamente los procesos y patrones biogeográficos que influyeron en 
la distribución actual de las especies. Entre algunas de las clasificaciones más recientes de la flora de los bosques secos del Ecuador se destacan los aportes de Aguirre et al. (2006a, 2006b), quienes establecen un sistema de clasificación de las formaciones vegetales basándose en la clasificación propuesta por Sierra (1999). El sistema propuesto por Aguirre y sus colaboradores es de gran interés para el desarrollo de estudios biogeográficos en los bosques secos de Ecuador y Perú.

Aquí presentamos un análisis panbiogeográfico para contribuir al conocimiento de los patrones de distribución de la flora de cactáceas del país.

\section{MATERIALES Y MÉTODOS}

Los datos sobre la distribución de las especies seleccionadas (Fig. 1-8) se obtuvieron a partir de la información recopilada en un estudio anterior (Loaiza et al. 2009), la cual se encuentra sistematizada en una base de datos sobre las colecciones de cactáceas presentes en los principales herbarios del Ecuador (QAP, QCA, QCNE y LOJA), así como también en algunas bases de datos de herbarios internacionales, como el de la Universidad de Aarhus (AAU) y el del Missouri Botanical Garden (MO), entre otros. Las especies seleccionadas fueron escogidas basados en dos tipos de criterios: a) Distribución conocida en los bosques secos del Ecuador, y b) Número de registros. Se descartó las especies con bajo número de registros y cuya distribución aún no se encuentra plenamente conocida. La clasificación propuesta en el presente trabajo fue debidamente analizada y elaborada en base a los resultados obtenidos en la presente investigación y en base a las clasificaciones de trabajos previos (Valencia 1999, Aguirre et al. 2006a, 2006b, Morrone 2006, Loaiza et al. 2009).

El enfoque panbiogeográfico enfatiza la importancia de la dimensión espacial (geográfica) de la biodiversidad, para permitir una comprensión más adecuada de los patrones y procesos evolutivos, destacando la relevancia de las distribuciones geográficas como objetos directos de análisis (Croizat 1958, 1964). El análisis básicamente consiste en marcar en mapas las localidades de distribución de distintas especies o grupos monofiléticos y luego se unen las localidades de registro de las especies mediante líneas de distancia menor, las cuales se denominan trazos individuales (Morrone \& Crisci 1990, Espinosa et al. 2002, Zunino \& Zullini 2003, Morrone 2004). Una vez obtenidos los trazos individuales, se superponen y cuando varios coinciden se considera un trazo generalizado, el cual permite sostener la existencia de una biota ancestral distribuida ampliamente y fragmentada por algún evento vicariante. Cuando dos o más trazos generalizados confluyen en una misma área, ésta se considera un nodo o área compuesta, lo cual implica a su vez un origen biótico múltiple, posiblemente en tiempos geológicos diferentes. Los nodos son áreas con una alta biodiversidad, las cuales debido principalmente a su mezcla biológica resultan prioritarias para la conservación (Espinosa et al. 2002, Morrone 2004, 2009).

\section{RESULTADOS}

La distribución geográfica y trazos individuales de las especies de cactáceas utilizadas en el presente análisis se muestran en las Figuras 1-8. El trazo de Armatocereus cartwrightianus (Britton \& Rose) Backeb. ex A.W. Hill (Fig. 1a) se encuentra en las provincias de El Oro, Guayas, Loja, Manabí y Santa Elena. Esta especie también se encuentra presente en los Departamentos de Tumbes, Piura y Lambayeque, en Perú (Ostolaza 2006). El trazo de Cleistocactus sepium var. morleyanus (Britton \& Rose) Madsen (Fig. 1b) se encuentra en las provincias de Chimborazo, Azuay y Cañar. El trazo de Opuntia cylindrica (Lam.) DC. (Fig. 2a) se encuentra en las provincias de Chimborazo, Cotopaxi, Pichincha, Cañar y Tungurahua. Esta especie también ha sido registrada en el norte del Perú (Madsen 1989). El trazo de Armatocereus laetus (Kunth) Backeb. ex A.W. Hill (Fig. 2b) se encuentra situado en la provincia de Loja. Esta especie también se encuentra presente en el Departamento de Piura, Perú (Ostolaza 2006). El trazo de Opuntia soederstromiana (Fig. 3a) se encuentra situado en las provincias de Chimborazo, Cotopaxi, Imbabura, Manabí, Pichincha y Esmeraldas. El trazo de Cleistocactus icosagonus (Kunth) F.A.C. Weber (Fig. 3b) se encuentra en las provincias de Azuay y Loja. Esta especie también se encuentra presente en el norte del Perú (Madsen 1989). El trazo de Opuntia quitensis F.A.C. Weber (Fig. 4) se encuentra en las provincias de Azuay, Chimborazo, El Oro, Loja, Pichincha, con un único registro en la provincia de Cotopaxi. Esta especie posee una amplia distribución y se encuentra presente en el norte de Perú (Madsen 1989) y en algunas zonas de Colombia (FernándezAlonso \& Estupiñán 2005). El trazo de Pilosocereus tweedyanus (Britton \& Rose) Byles \& G.D. Rowley (Fig. 5a) se encuentra en las provincias de Azuay, El Oro, Guayas, Loja, Manabí y Santa Elena. Esta especie también ha sido registrada en el norte de Perú (Madsen 1989). El trazo de Cleistocactus sepium (Kunth) F.A.C. Weber var. sepium (Fig. $5 b)$ se encuentra presente en las provincias de Chimborazo, Cotopaxi y Tungurahua. El trazo de Cleistocactus sepium var. ventimigliae (Riccob.) Madsen (Fig. 6) tiene una distribución mucho más amplia que las dos variedades anteriores y se encuentra en las provincias de Azuay, Cañar, Carchi, Chimborazo, Pichincha, Imbabura y Esmeraldas. El trazo de Echinopsis pachanoi (Britton \& Rose) Friedrich \& G.D. Rowley (Fig. 7) se encuentra presente en las provincias de Azuay, Cañar, Chimborazo, Imbabura, Loja, Pichincha y Tungurahua. Esta especie también se encuentra presente en el norte de Perú (Madsen 1989) y en Colombia, en el 


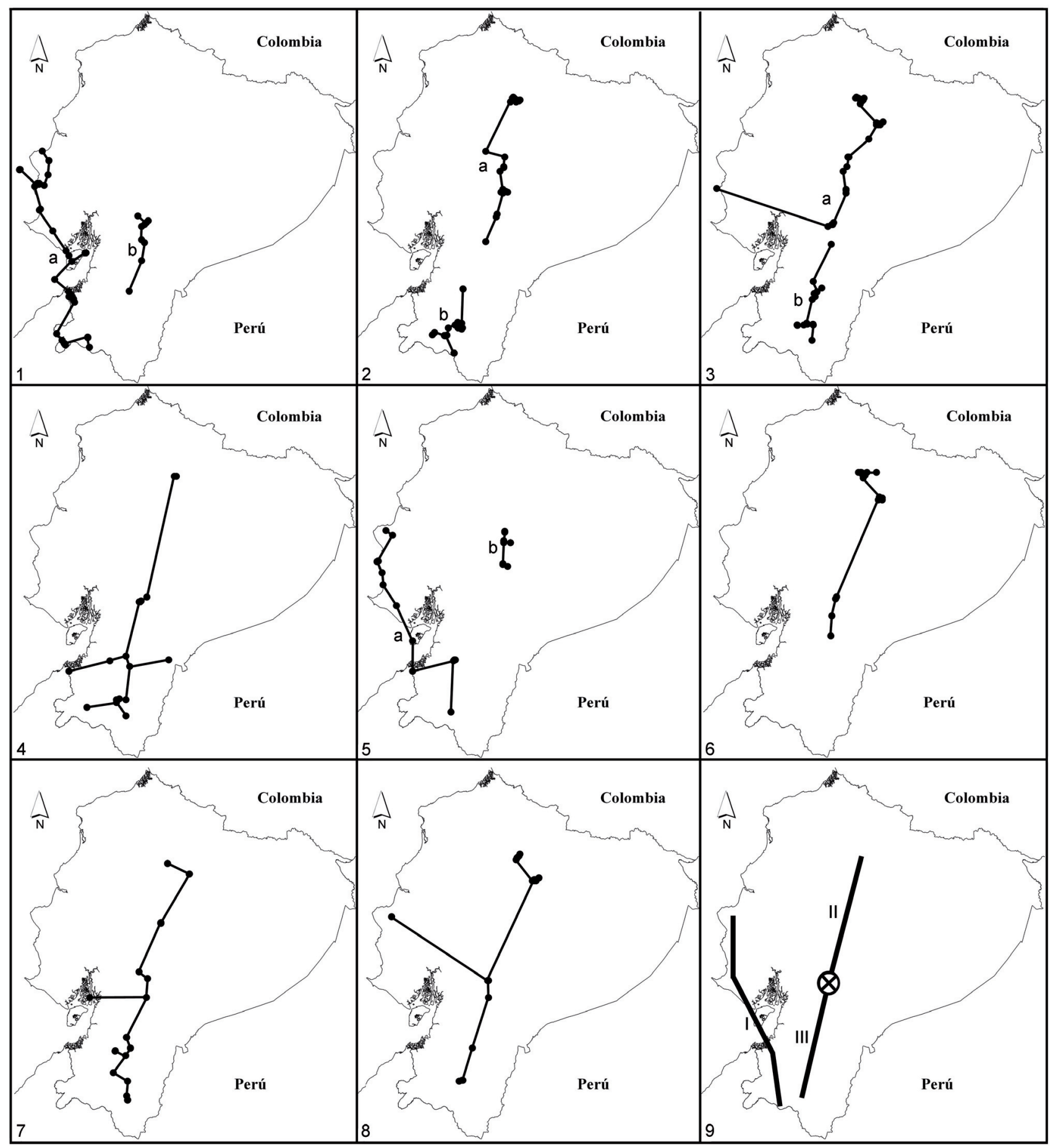

Figuras 1-9. Trazos individuales, generalizados y nodos. 1-8, Trazos individuales: 1a Armatocereus cartwrightianus; 1b, Cleistocactus sepium var. morleyanus; 2a, Opuntia cylindrica, 2b, A. laetus; 3a, O. soederstromiana, 3b, C. icosagonus; 4, O. quitensis; 5a, Pilosocereus tweedyanus, 5b, C. sepium var. sepium; 6, C. sepium var. ventimigliae; 7, Echinopsis pachanoi; 8, O. pubescens; 9, trazos generalizados y nodo.

FIGURES 1-9. Individual tracks, generalized and nodes. 1-8, Individual tracks: 1a Armatocereus cartwrightianus; 1b, Cleistocactus sepium var. morleyanus; 2a, Opuntia cylindrica, 2b, A. laetus; 3a, O. soederstromiana, 3b, C. icosagonus; 4, O. quitensis; 5a, Pilosocereus tweedyanus, 5b, C. sepium var. sepium; 6, C. sepium var. ventimigliae; 7, Echinopsis pachanoi; 8, O. pubescens; 9, generalized tracks and node. 
Departamento de Nariño (Fernández-Alonso \& Estupiñán 2005). El trazo de Opuntia pubescens J.C. Wendl. ex Pfeiff. (Fig. 8) se distribuye en las provincias de Azuay, Cañar, Chimborazo, Imbabura, Loja, Pichincha y Manabí. Esta especie también se encuentra distribuida en el norte del Perú (Madsen 1989).

La superposición de los ocho trazos individuales obtenidos en el presente análisis permitió la identificación de tres trazos generalizados (Fig. 9): costero seco (I), interandino del centro-norte (II) e interandino del sur (III).

El trazo costero seco se encuentra situado en las provincias de Loja, El Oro, Guayas, Santa Elena y Manabí, y está sustentado por las especies Armatocereus cartwrightianus (Fig. 1a) y Pilosocereus tweedyanus (Fig. 5a). Otras especies que también están presentes dentro de este trazo son Opuntia quitensis (Fig. 4), O. soederstromiana (Fig. 3a), Echinopsis pachanoi (Fig. 7) y $O$. pubescens (Fig. 8). Este trazo generalizado se sitúa en las provincias biogeográficas del Ecuador Árido y Tumbes-Piura y comprende las formaciones vegetales de Bosque Seco Deciduo (de), Bosque Seco Semideciduo (sd) y Matorral Seco Espinoso (ms) (Madsen et al. 2001, Aguirre et al. 2006a, 2006b).

El trazo interandino del centro-norte se encuentra situado en las provincias de Imbabura, Pichincha, Napo, Cotopaxi, Tungurahua, Bolívar, Chimborazo y parte de la provincia del Cañar. Se encuentra sustentado por Cleistocactus sepium var. morleyanus (Fig. 1b), Opuntia cylindrica (Fig. 2a), O. soederstromiana (Fig. 3a), C. sepium var. sepium (Fig. 5b), O. quitensis (Fig. 4), C. sepium var. ventimigliae (Fig. 6), Echinopsis pachanoi (Fig. 7) y O. pubescens (Fig. 8). Este trazo generalizado se sitúa en la provincia biogeográfica del Occidente de Ecuador y comprende las formaciones vegetales de Bosque Seco Interandino del Norte (i-n) y Bosque Seco Montano Bajo (sm) (Aguirre et al. 2006a).

El trazo interandino del sur se encuentra situado en las provincias de Loja, Azuay y parte de la provincia del Cañar. Se encuentra sustentado por Armatocereus laetus (Fig. 2b), Cleistocactus icosagonus (Fig. 3b), Opuntia quitensis (Fig. 4), Echinopsis pachanoi (Fig. 7) у O. pubescens (Fig. 8). Otras especies que también se encuentran incluidas dentro de este trazo son Cleistocactus sepium var. morleyanus (Fig. 1b) y Pilosocereus tweedyanus (Fig. 5a). Este trazo generalizado se sitúa en la provincia biogeográfica del Occidente de Ecuador y comprende las formaciones vegetales de Bosque Seco Interandino del Sur (i-s) y Matorral Seco Espinoso (ms) (Aguirre et al. 2006a, Loaiza et al. 2009).

La superposición de los trazos generalizados interandino del sur e interandino del centro-norte permitió identificar la presencia de un nodo (Fig. 9). Este nodo se sitúa entre las provincias de Cañar y Chimborazo, en la provincia biogeográfica del Occidente de Ecuador.

\section{DISCUSIÓN}

La mayor parte de las especies de cactáceas registradas en el Ecuador se encuentran distribuidas en un área situada entre el noroccidente del Perú y el centro y suroccidente del país (Madsen 1989). Pocas especies tienen un rango de distribución amplio, entre las que se incluyen varias epifitas (Madsen 1989, 2002, Loaiza et al. 2009) y algunas otras especies como Echinopsis pachanoi y Opuntia quitensis (Fernández-Alonso \& Estupiñán 2005). El género Opuntia se caracteriza por ser uno de los géneros de cactáceas con la mayor distribución a nivel del continente (Britton \& Rose 1919, Anderson 2001), por lo cual muchas de las especies presentes en el Ecuador podrían haber tenido una distribución mucho más amplia en la antigüedad. Sin embargo, esta distribución estaría claramente determinada por la efectividad de los mecanismos de dispersión de ciertas especies, tal es el caso de Opuntia quitensis y $O$. pubescens, cuya facilidad para el desprendimiento de los segmentos de su tallo les ha permitido tener una distribución bastante favorable. Por otra parte, la presencia de barreras geográficas habría sido la principal limitante para la expansión del área de distribución de muchas especies, esto permitiría explicar la presencia de O. quitensis en Colombia y la total ausencia de otras especies. El caso de Echinopsis pachanoi difiere del resto de las especies, debido a que ha sido utilizada desde tiempos ancestrales por el ser humano en Perú y Ecuador y es muy probable que haya sido introducida en Colombia y en ciertas zonas de la costa ecuatoriana por alguna cultura ancestral (Madsen et al. 2001).

El nodo situado en la superposición de los trazos generalizados interandino del sur e interandino del centronorte ayudaría a entender y explicar la multiplicidad de orígenes de los linajes allí presentes y la gran diferencia entre las especies de cactáceas presentes en ambos trazos generalizados, muchas de las cuales son endémicas para cada región. Algunas de las especies propiamente originadas en el nodo identificado y que constituirían el trazo interandino del centro-norte serían las siguientes: Armatocereus godingianus, Cleistocactus leonensis, $C$. sepium (tres variedades), Espostoa frutescens, Opuntia aequatorialis Britton \& Rose, O. bakeri Madsen, $O$. soederstromiana y Weberocereus rosei (Kimnach) Buxb.

Algunos autores, como Valencia et al. (1999) en Sierra (1999), también han coincidido en diferenciar los valles secos interandinos del centro-norte de los de la región sur (Loja). Al norte del país, los valles son más altos y se encuentran ubicados en rangos altitudinales entre 1.800$2.600 \mathrm{~m}$. Estos valles son más aislados debido a que ambas faldas (orientales y occidentales) están cubiertas con bosques montanos muy húmedos. Por el contrario, en la región sur las faldas occidentales son relativamente más secas y las montañas más bajas, ocasionando que los 
bosques secos interandinos se encuentren ubicados desde los $1.300 \mathrm{~m}$ hacia arriba, lo que probablemente facilita un mayor intercambio entre los bosques secos de la costa y los bosques secos interandinos (Aguirre et al. 2006a). La existencia de estos dos trazos generalizados dentro de una provincia biogeográfica podría permitir en un futuro su reconocimiento como distritos. Sin embargo, es necesario señalar que únicamente el trazo interandino del sur tendría una distribución prolongada hasta ciertas zonas del norte de Perú, siendo probablemente interrumpido por la presencia de barreras geográficas que habrían limitado la libre distribución de las especies, promoviendo en algunos casos la formación de subespecies dentro de un contexto vicariante, tal es el caso de Melocactus bellavistensis Rauh \& Backeb. subsp. bellavistensis (Loaiza 2010). Otros géneros en cambio (Armatocereus y Browningia), cuyo origen se atribuye a los bosques secos del norte de Perú, deberían ser analizados conjuntamente con otros géneros endémicos de dicho país, a fin de establecer la probable existencia de algún nodo no identificado, lo cual ayudaría a entender claramente los procesos de distribución geográfica de ciertas especies.

Por otra parte, el nodo obtenido en la superposición de los trazos generalizados interandino del sur e interandino del centro-norte del Ecuador, sería de gran importancia para el establecimiento de una zona prioritaria para la conservación de cactáceas, dado que combina representantes de dos componentes bióticos diferentes, además de que su importancia también podría ser considerada para la conservación de otros grupos de plantas con patrones de distribución geográfica similares.

\section{AGRADECIMIENTOS}

La presente investigación recibió el apoyo del Instituto de Ecología y del Herbario (HUTPL) de la Universidad Técnica Particular de Loja. Se desea agradecer la colaboración de las siguientes personas e instituciones: Carlos Cerón (QAP), Katya Romoleroux (QCA), Elsa Toapanta (QCNE) y Zhofre Aguirre del Herbario LOJA por permitir el acceso y revisión de las colecciones a su cargo. Se agradece también las sugerencias y valiosos comentarios realizados por dos revisores anónimos.

\section{BIBLIOGRAFÍA}

Aguirre, Z., L.P. Kvist \& O. SÁnchez. 2006a. Bosques secos en Ecuador y su diversidad. En: M. Moraes, B. Øllgaard, L. P. Kvist, F. Borchsenius \& H. Balslev (eds.), Botánica Económica de los Andes Centrales. Pp. 162-187. Universidad Mayor de San Andrés, La Paz.

Aguirre, Z., R. Linares-Palomino \& L.P. Kvist. 2006 b. Especies leñosas y formaciones vegetales en los bosques estacionalmente secos de Ecuador y Perú. Arnaldoa 13(2): 324-350.

Anderson, E.W. 2001. The cactus family. O. R. Timber Press, Portland. $776 \mathrm{pp}$.

Bravo, H. 1978. Las cactáceas de México. Vol. I. Universidad Nacional Autónoma de México. México, D.F. 743 pp.

Britton, N.L. \& J.N. Rose. 1919. The Cactaceae: Descriptions and illustrations of plants in the cactus family. Vol. 1. The Carnegie Institution of Washington. Washington D.C. 236 pp.

Britton, N.L. \& J.N. Rose. 1920. The Cactaceae: Descriptions and illustrations of plants in the cactus family. Vol. 2. The Carnegie Institution of Washington. Washington D.C. 241 pp.

Croizat, L. 1958. Panbiogeography. Vols. 1 y 2. Publicado por el autor, Caracas. 1731 pp.

Crolzat, L. 1964. Space, time, form: The biological synthesis. Publicado por el autor, Caracas. $889 \mathrm{pp}$.

Espinosa, D., J.J. Morrone, J. Llorente \& O. Flores. 2002. Introducción al análisis de patrones en biogeografía histórica. Las Prensas de Ciencias, Facultad de Ciencias, UNAM. México, D. F. 133 pp.

Fernández-Alonso, J.L. \& C. EstupiñÁn. 2005. Echinopsis pachanoi y Opuntia quitensis, dos cactáceas nuevas para la flora de Colombia. Boletín de la Sociedad Latinoamericana y del Caribe de Cactáceas y otras Suculentas 2(3): 3-4.

Loaiza, C.R., Z.H. Aguirre \& O. JadÁn. 2009. Estado del conocimiento actual de la familia Cactaceae en el Ecuador. Boletín de la Sociedad Latinoamericana y del Caribe de Cactáceas y otras Suculentas 6(3): 11-22.

LoAizA, C.R. 2010. Análisis biogeográfico de Melocactus bellavistensis en la región sur del Ecuador: aspectos taxonómicos, distribución y conservación. Cinchonia 10(1): 106-118.

Madsen, J.E. 1989. Cactaceae. En G. Harling y L. Anderson (eds.), Flora of Ecuador 35: 1-79.

Madsen, J.E. 2000. Fitogeografía de las cactáceas del Ecuador. Memorias del Tercer Congreso Ecuatoriano de Botánica. Quito. Pp. 21-22.

Madsen, J.E. 2002. Cactus en el sur del Ecuador. En: Z. Aguirre et al., Botánica Austroecuatoriana: Estudios sobre los Recursos Vegetales en las Provincias de El Oro, Loja y Zamora Chinchipe, pp. 289-303. Primera Edición. Ediciones AbyaYala. Quito.

Madsen, J.E., R. Mix \& H. Balslev. 2001. Flora of Puna Island: Plant resources on a Neotropical island. Aarhus University Press. 289 pp.

Morrone, J.J. 2004. Panbiogeografía, componentes bióticos y zonas de transición. Revista Brasileira de Entomología 48(2): 149-162.

Morrone, J.J. 2006. Biogeographic areas and transition zones of Latin America and the Caribbean Islands based on panbiogeographic and cladistic analyses of the entomofauna. Annual Review of Entomology 51: 467-494.

Morrone, J.J. 2009. Evolutionary biogeography: An integrative approach with case studies. Columbia University Press, Nueva York, $301 \mathrm{pp}$.

Morrone, J.J. \& J.V. Crisci. 1990. Panbiogeografía: Fundamentos y Métodos. Evolución Biológica 4: 119-140.

Ostolaza, C. 2006. El género Armatocereus Backeberg. Zonas Áridas 10: 144-154.

Sierra, R. (ed.). 1999. Propuesta preliminar de un sistema de 
clasificación vegetal para el Ecuador Continental. Proyecto INEFAN / GEF-BIRF y Ecociencia. Quito. 193 pp.

UlloA, C. \& D.A. NeIll. 2005. Cinco años de adiciones a la flora del Ecuador: 1999-2004. Missouri Botanical Garden / Herbario Nacional del Ecuador. Quito. 75 pp.

Valencia, R., C. Cerón, W. Palacios \& R. Sierra. 1999. Las formaciones naturales de la Sierra del Ecuador. En: R. Sierra (ed.), Propuesta Preliminar de un Sistema de Clasificación Vegetal para el Ecuador Continental. Pp. 79-108. Proyecto
INEFAN / GEF-BIRF y EcoCiencia, Quito.

Valencia, R.N., N.S. Pitman, S. León-Yánez \& P.M. Jorgensen (eds.). 2000. Libro Rojo de las Plantas Endémicas del Ecuador. Herbario QCA / Pontificia Universidad Católica del Ecuador. Quito. $489 \mathrm{pp}$.

Zunino, M. \& A. Zullins. 2003. Biogeografía: La dimensión espacial de la evolución. Fondo de Cultura Económica, México, D.F. 359 pp.

Recibido: 22.02.11

Aceptado: 06.08.11 